15

\title{
Исследование радиационной эрозии в газоразрядном детекторе с помощью атомно-силовой микроскопии
}

\author{
(C) М.Э. Бузоверя, ${ }^{1}$ Г.Е. Гаврилов, ${ }^{2, \text { O.Е. Маев }}{ }^{2}$ \\ ${ }^{1}$ Саровский ффизико-технический институт НИЯУ МИФИ, \\ 607186 Саров, Россия \\ ${ }^{2}$ Петербургский институт ядерной физики НИЦ „Курчатовский институт“, \\ 188300 Санкт-Петербург, Гатчина, Россия \\ ฯ e-mail: gavrilov_ge@pnpi.nrcki.ru
}

Поступило в Редакцию 23 июля 2020 г.

В окончательной редакции 27 августа 2020 г.

Принято к публикации 7 сентября 2020 г.

Методами атомно-силовой микроскопии исследованы образцы катодов многопроволочных пропорциональных камер, подвергнутых в ходе лабораторных ресурсных испытаний длительному облучению $\beta$-источником ${ }^{90} \mathrm{Sr}$. Описаны изменения морфологии поверхности медной фольги на катоде детектора, произошедшие в результате воздействия потока электронов. Представлены количественная оценка и анализ эволюции радиационных дефектов в зависимости от дозы облучения. Продемонстрировано сходство радиационных дефектов в лабораторных прототипах и в полномасштабных пропорциональных камерах, отработавших 10 лет на Большом адронном коллайдере.

Ключевые слова: атомно-силовая микроскопия, газоразрядные детекторы, радиационные дефекты, окислы меди, блистеры, кратеры, эффект Мальтера.

DOI: 10.21883/JTF.2021.02.50374.234-20

\section{Введение}

Целью настоящей работы является демонстрация эффективности методов атомно-силовой микроскопии (ACM) при изучении причин возникновения спонтанных самоподдерживающихся токов в многопроволочных пропорциональных камерах. Впервые исследование образцов поверхности катодов газоразрядных детекторов, работающих в экспериментах на Большом адронном коллайдере (БАК), выполнено с широким использованием методов АСМ. Объектом изучения стали образцы катода, взятые из лабораторных прототипов многопроволочных стриповых пропорциональных камер (Cathode Strip Chambers - CSC) эксперимента CMS.

Первые ресурсные испытания пропорциональных камер CSC в начале 2000-х годов выполнялись в ПИЯФ (Петербургском институте ядерной физики) и в ЦЕРН (Европейский центр ядерных исследований) как с помощью прототипов, так и полномасштабных пропорциональных камер $[1,2]$. По результатам работ была выполнена оптимизация состава рабочей газовой смеси и материалов конструкции. В результате пропорциональные камеры показали надежность и высокую радиационную стойкость при многолетней работе в эксперименте на БАК.

Сегодня интерес к исследованиям радиационной стойкости газоразрядных детекторов, работающих на БАК, снова вырос. Это связано с планируемым на 2025-й год почти десятикратным увеличением светимости БАК и, как следствие, с необходимостью обеспечить радиационную стойкость детекторов на последующие 10 лет работы. Важной мотивацией выполненной работы стало периодическое появление в пропорциональных камерах спонтанных самоподдерживающихся токов (мальтеровский эффект (МЭ)) [3], которые в десятки раз превышают ток ионизации в эксперименте. Спонтанные токи до 30-40 $\mu \mathrm{A}$ вызывают ложные срабатывания детекторов и ускоренное старение анодных проволочек в области генерации МЭ.

Визуальный осмотр после лабораторных ресурсных испытаний как разобранных прототипов, так и полномасштабных камер показал, что характерным результатом длительного облучения электронами является окисление медной фольги на катоде. Источником появления кислорода в рабочей газовой смеси пропорциональных камер является обычно входящий в ее состав углекислый газ - $\mathrm{CO}_{2}$. Энергия электронов в лавине у поверхности анодной проволочки достигает на краю энергетического распределения $E_{e} \sim 50 \mathrm{eV}$. Поэтому в газоразрядной плазме лавины происходят реакции диссоциативной ионизации молекул $\mathrm{CO}_{2}$ [4]. Вблизи анода образуются радикалы $\mathrm{O}^{\bullet}$ и $\mathrm{CO}^{\bullet}(\bullet-$ число непарных электронов на внешней оболочке) и молекулы кислорода $\mathrm{O}_{2}$, дрейфующие к катоду. В результате поверхность катодной медной фольги окисляется. Процесс окисления в первую очередь развивается на стыках микрозерен, из которых состоит кристаллическая структура меди, размерами от сотен нанометров до десятков микрон, прочно связанных между собой по контактным поверхностям [5].

Очевидным следствием появления оксидных образований является возрастание механического напряжения 
поверхности медной фольги. Это связано с разницей плотностей чистой меди $\left(\rho_{\mathrm{Cu}}=8.92 \mathrm{~g} \cdot \mathrm{cm}^{-3}\right)$ и образующихся в приповерхностном слое на межзеренных стыках гранул окислов $\mathrm{Cu}_{2} \mathrm{O}\left(\rho_{\mathrm{Cu}_{2} \mathrm{O}}=6.0 \mathrm{~g} \cdot \mathrm{cm}^{-3}\right)$ и $\mathrm{CuO}\left(\rho_{\mathrm{CuO}}=6.31 \mathrm{~g} \cdot \mathrm{cm}^{-3}\right)$. Возникающие напряжения в кристаллической структуре металла приводят к появлению дефектов поверхности и росту шероховатости. Известно, что оксид $\mathrm{CuO}$ обладает удельным сопротивлением $0.01-1 \Omega \cdot \mathrm{cm}$, в то время как для оксида $\mathrm{Cu}_{2} \mathrm{O}$ удельное сопротивление может варьироваться в диапазоне $10^{3}-10^{8} \Omega \cdot \mathrm{cm}[6]$. В работах $[7,8]$ показано, что гранулы высокорезистивных окислов $\mathrm{Cu}_{2} \mathrm{O}$ в электрическом поле порядка $50000 \mathrm{~V} \cdot \mathrm{cm}^{-1}$, заряжаясь положительными ионами из газоразрядной плазмы, являются источником ультрафиолетовых фотонов и электронов вторичной эмиссии даже без наличия значительных дефектов поверхности катода.

Кроме процессов окисления, на морфологию поверхности катода в пропорциональной камере влияет и радиационная эрозия, вызванная взаимодействием электронов с энергией от 0.5 до $2.28 \mathrm{MeV}$ с медной фольгой. Воздействие электронов инициирует в металле целый комплекс физических процессов: нагрев, плавление, распространение ударных волн, пластическую деформацию, структурные превращения. Появляются блистеры, кратеры, флекинг и микропики, которые с увеличением дозы преобразуются в поры. В итоге радиационная эрозия приводит к послойному разрушению поверхности $[9,10]$. Природа развития этих дефектов исследована и описана в работах $[11,12]$.

Усредненная напряженность электрического поля на поверхности катодных плоскостей пропорциональных камер составляет $\sim 5000 \mathrm{~V} \cdot \mathrm{cm}^{-1}$. В результате дефекты в виде микропиков высотой $h \sim 2-3 \mu \mathrm{m}$, концентрируя на остриях диаметром $d \sim 0.01-0.1 \mu$ с силовые линии электрического поля, имеют напряженность поля в десятки раз выше - пропорционально величине $k \propto h / d$. Это приводит к понижению работы выхода электронов с поверхности на остриях. Вероятность эмиссии электронов усиливается из-за абсорбции на поверхности микропиков положительных ионов из газовой смеси. В результате микропики, где на остриях достигается напряженность поля $\geq 1 \cdot 10^{6} \mathrm{~V} \cdot \mathrm{cm}^{-1}$, способны стать источниками вторичной электронной эмиссии, инициирующей появление МЭ [7].

Поэтому основными объектами АСМ анализа образцов катода пропорциональных камер, прошедших ресурсные испытания, стали образовавшиеся микропики и гранулы окислов меди.

\section{1. Экспериментальная часть}

\section{1. Условия облучения образцов в ресурсных испытаниях}

Для облучения прототипов пропорциональных камер CSC в ходе ресурсных испытаний использовал- ся $\beta$-источник ${ }^{90} \mathrm{Sr}\left(E_{\beta}^{\max }=2.28 \mathrm{MeV}\right)$. Энергетический спектр электронов стронция перекрывает энергию электронов фона в пропорциональных камерах экспериментальных установок, составляющую около $1 \mathrm{MeV}$, поэтому при проведении ресурсных испытаний предполагалось, что характер радиационной эрозии в прототипах на стенде должен совпадать с эрозией медной фольги в пропорциональных камерах CSC. Важно отметить, что большая часть энергетического спектра $\beta$-источника превышает пороговый уровень образования радиационных дефектов в металлах $\sim 0.5 \mathrm{MeV}[13]$.

Плотность потока электронов при облучении прототипа в первом цикле старения составила $F \approx 4.36 \cdot 10^{6} \mathrm{~s}^{-1} \cdot \mathrm{cm}^{-2}$ и обеспечила в детекторе ток ионизации $17 \mu \mathrm{A}$. Целью этого испытания было получение верхней оценки радиационной стойкости пропорциональной камеры с рабочей газовой смесью $40 \% \mathrm{Ar}+50 \% \mathrm{CO}_{2}+10 \% \mathrm{CF}_{4}$. За время старения $2.1 \cdot 10^{6} \mathrm{~s}$ через газовый объем прототипа прошел заряд электронов $1.36 \mathrm{C} \cdot \mathrm{cm}^{-1}$, что, согласно оценкам, соответствует заряду, накопленному пропорциональной камерой за 50 лет работы на БАК. Флюенс электронов в испытании составил $1 \cdot 10^{13} \mathrm{~cm}^{-2}$. При этом прототип CSC сохранил свои рабочие характеристики и не показал никаких проявлений Мальтер-эффекта.

Во втором цикле старения скорость аккумулирования заряда в прототипе была уменьшена более чем в 4 раза за счет установки перед излучателем медного фильтра, в результате чего ток ионизации составил $4 \mu \mathrm{A}$. В прототипе использовалась модифицированная газовая смесь $36.6 \% \mathrm{Ar}+61.7 \% \mathrm{CO}_{2}+1.7 \% \mathrm{CF}_{4}$. Испытание проводилось для изучения устойчивости к старению прототипа CSC с газовой смесью, в которой содержание фреона $\mathrm{CF}_{4}$ уменьшено в 5 раз по сравнению с рабочей смесью. Аккумулированный заряд за время $2.3 \cdot 10^{6} \mathrm{~s}$ во втором цикле старения составил $0.39 \mathrm{C} \cdot \mathrm{cm}^{-1}$. Флюенс электронов в этом испытании составил $2 \cdot 10^{12} \mathrm{~cm}^{-2}$. Деградации рабочих характеристик камеры в этом случае также не было обнаружено.

Отсутствие проявления МЭ в лабораторных ресурсных испытаниях легко объяснимо разницей в габаритах и в длительности облучения лабораторных прототипов и полномасштабных камер. Так, длительность работы камер CSC на сегодня составляет около $1 \cdot 10^{8} \mathrm{~s}$, а общая их площадь $\sim 1000 \mathrm{~m}^{2}$. Для сравнения площадь облучения прототипа $\sim 900 \mathrm{~cm}^{2}$.

Содержание углекислого газа в газовых смесях во время ресурсных испытаний составило $\sim 60 \%$. Это обеспечило условия, при которых скорость образования кислородосодержащих молекул и ионов в газе зависела только от плотности потока электронов.

На рис. 1 (вверху) показана схема лабораторного прототипа пропорциональной камеры CSC. Детектор состоит из трех прямоугольных катодных панелей $30 \times 30 \mathrm{~cm}$, разделенных двумя зазорами по $0.9 \mathrm{~cm}$, через которые продувалась газовая смесь $\mathrm{Ar} / \mathrm{CO}_{2} / \mathrm{CF}_{4}$. Катодные панели 

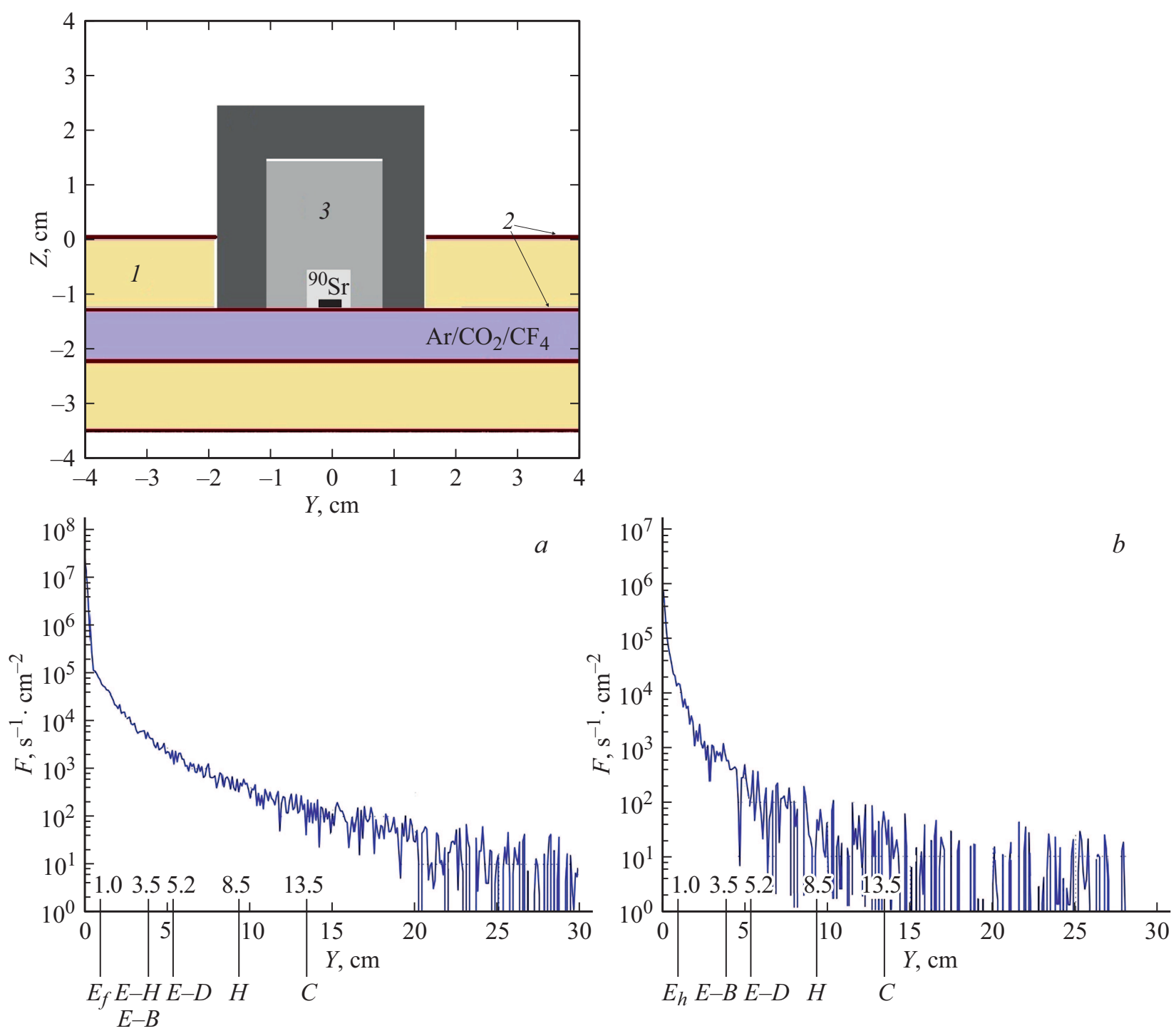

Рис. 1. Вверху - схема облучения $\beta$-источником ${ }^{90} \mathrm{Sr}$ при Монте-Карло симуляции: 1 - сотовый поликарбонат (honeусотb) толщиной $1.6 \mathrm{~cm} ; 2-$ фольгированный стеклопластик FR4 толщиной $0.15 \mathrm{~cm} ; 3-{ }^{90} \mathrm{Sr}$ в пластиковом контейнере; $4-$ свинцовая защита источника. Внизу: $a$ - распределение $F, \mathrm{~s}^{-1} \cdot \mathrm{cm}^{-2}$ - плотности потока электронов в зависимости от расстояния до центра зоны облучения $(\mathrm{Y}=0 \mathrm{~cm})$ для первого цикла старения. $b-$ распределение $F, \mathrm{~s}^{-1} \cdot \mathrm{cm}^{-2}$ во втором цикле старения. Выносными линиями на оси координат указаны расстояния от центра зоны облучения до исследуемых образцов катода.

были изготовлены из пластин поликарбоната с сотовой гексагональной структурой (honeycomb) толщиной $1.6 \mathrm{~cm} \mathrm{1.} \mathrm{Пластины} \mathrm{заключены} \mathrm{между} \mathrm{листами} 0.15 \mathrm{~cm}$ фольгированного стеклопластика FR4 2. Толщина медной фольги $35 \mu \mathrm{m}$. После каждого цикла старения для исследования из катодных панелей выпиливались образцы фольгированного стеклопластика в форме дисков диметром $2.3 \mathrm{~cm}$. Для облучения использовался $\beta$-источник ${ }^{90} \mathrm{Sr}$, установленный на верхнем катоде прототипа в пластиковом контейнере 3, окруженном свинцовой защитой 4 (рис. 1, вверху).

С целью оценки распределения радиационной загрузки от источника было выполнено компьютерное моделирование прохождения ионизирующего излучения от источника ${ }^{90} \mathrm{Sr}$. Энергетический спектр бета-частиц и фотонов, образующихся в источнике, моделировался согласно данным работы [13]. Прохождение ионизирующего излучения через прототип и защитный контейнер источника моделировалось при помощи программного пакета GEANT4 [14].

На графиках на рис. 1 показаны распределения плотности потока электронов $F$ при облучении в первом (слева) и втором (справа) циклах старения в зависимости от расстояния до источника ${ }^{90} \mathrm{Sr}(Y=0 \mathrm{~cm})$. Видно, что в обоих циклах наибольшая плотность потока $F \geq 1 \cdot 10^{4} \mathrm{~s}^{-1} \cdot \mathrm{cm}^{-2}$ сосредоточена в зоне с радиусом $Y \approx 2.0 \mathrm{~cm}$. С удалением от ее центра плотность потока быстро спадает до $2 \cdot 10^{1}-1 \cdot 10^{2} \mathrm{~s}^{-1} \cdot \mathrm{cm}^{-2}$ по всей остальной площади катода. Вдоль оси координат на обоих графиках рис.1 выносными линиями указаны рас- 

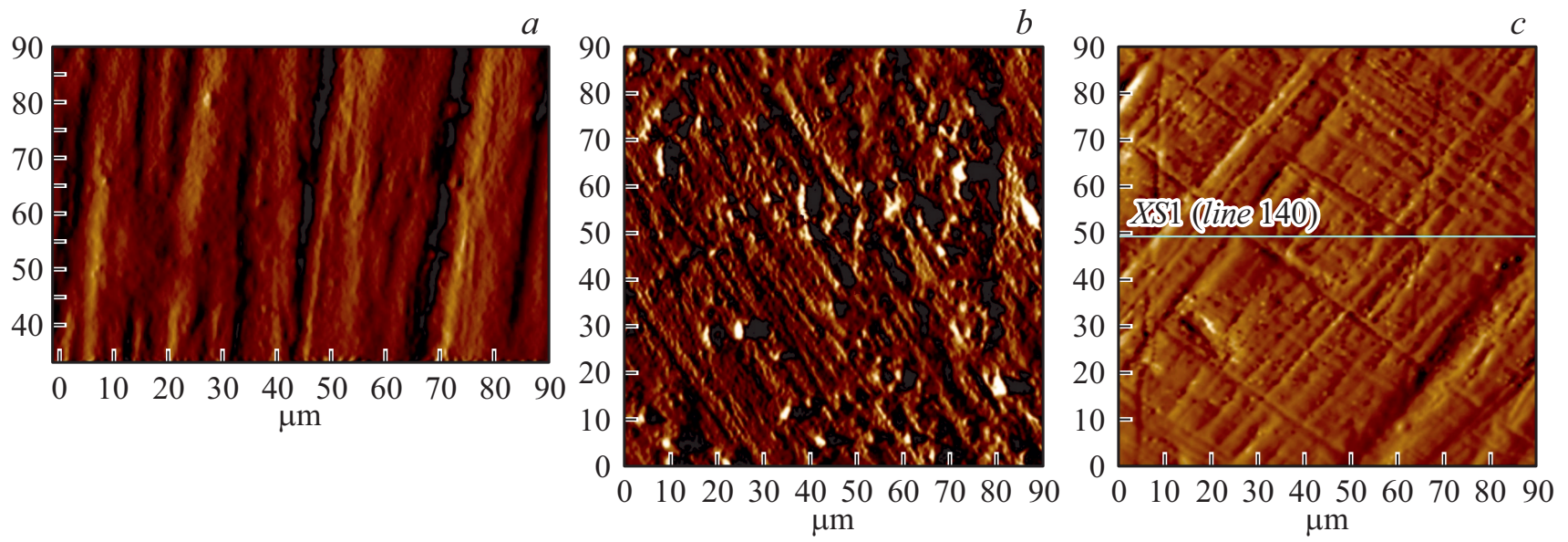

Рис. 2. АСМ изображения, поле $90 \times 90 \mathrm{~mm}: a-$ образец сравнения; $b-$ серия 1 , образец $H$, доза $D=8 \mathrm{mGy} ; c-$ серия 2 , образец $H$, доза $D=0.2 \mathrm{mGy}$.

стояния от центра зоны облучения до образцов, взятых с поверхности катода для анализа. На рис. 1, а внизу показано, что в первом цикле старения (серия 1) из плоскости катода, были взяты образцы фольгированного стеклопластика в точках $E_{f}(1 \mathrm{~cm}), E-H(3.5 \mathrm{~cm}), E-B$ $(3.5 \mathrm{~cm}), H(8.5 \mathrm{~cm})$ и $C(13.5 \mathrm{~cm})$. Аналогично после второго цикла старения (серия 2) были взяты образцы: $E_{h}(1 \mathrm{~cm}), E-B(3.5 \mathrm{~cm}), E-D(5.2 \mathrm{~cm}), H(8.5 \mathrm{~cm})$ и $C(13.5 \mathrm{~cm})$ (рис. $1, b)$. Для контрольных измерений использовался образец сравнения, вырезанный из листа стеклопластика FR4. На основании приведенных выше распределений интенсивности облучения были выполнены расчеты интегральных доз D (размерностью в милигреях $(\mathrm{mGy}))$ для каждого образца поверхности катода [15]. Эти данные приводятся далее.

\section{2. Приборы и методы измерений}

Исследования морфологии поверхности образцов методом АCM осуществлялись на C3M ,Solver Next“ производства ОАО „НТ-МДТ“ г. Зеленоград. Сканирование поверхности проводилось на воздухе при нормальных условиях кантилеверами серии $\mathrm{NSG10} / \mathrm{W}_{2} \mathrm{C}$ полуконтактным методом (tapping-mode) в режимах топографии и фазового контраста. В первом режиме для восстановления рельефа поверхности используется модуляция амплитуды сигнала кантилевера. Во втором, для получения рельефа, измеряется величина сдвига фазы колебаний кантилевера, которая зависит от плотности материала в точке сканирования. Поэтому именно режим фазового контраста дает информацию о микронеоднородностях свойств поверхностных слоев образца. Результаты сканирования обрабатывались программным модулем обработки изображения Image Analysis 3.5.

\section{2. Результаты и обсуждение}

\section{1. Морфология поверхности образцов}

На рис. 2, а показана поверхность фольгированного стеклопластика FR4 образца сравнения. Необлученная поверхность фольги имеет волокнистую структуру и представляет собой чередование гребней и впадин. Это связано с технологией производства FR4, когда медная фольга под давлением при температуре около $130^{\circ} \mathrm{C}$ приклеивается к листу стеклопластика. В результате на поверхности фольги проявляется структура стекловолокон.

На рис. 2, b, $c$ представлены поверхности образцов катода $H$, равноудаленных от центра облучения $(Y=8.5 \mathrm{~cm})$ из 1-й и 2-й серий. Видно, что состояние поверхности после ресурсных испытаний кардинально отличается от состояния до облучения. Особенно хорошо прослеживаются радиационные дефекты на образце с бо́льшей дозой облучения (рис. $2, b$ ).

В образце 1-й серии $H$ после аккумулирования дозы $D \approx 8 \mathrm{mGy}$ исчезла типичная для исходной фольгированной меди волнистость, отражающая профиль стекловолокон. Поверхность фольги стала бугристой и шероховатой. Радиационная эрозия здесь проявилась в виде эффектов блистеринга, флекинга, кратерообразования и за счет распыления вещества.

На рис. 2, с видно, что у образца 2-й серии $H$ поверхность фольги при дозе $D \approx 0.2 \mathrm{mGy}$ стала тонковолокнистой. Количество мелких блистеров в виде маленьких пузырьков заметно увеличилось по сравнению с необлученным образцом сравнения (рис. 2,a). При большом разрешении с полем изображения $90 \times 90 \mu \mathrm{m}$ трудно выделить отдельные структурные элементы, поэтому для детализации структуры были выполнены малоразмерные сканы $10 \times 10 \mu \mathrm{m}$.

На рис. 3 выделены агрегаты из трех структурных элементов (CЭ) в образце серии 2, удаленном от центра зо- 

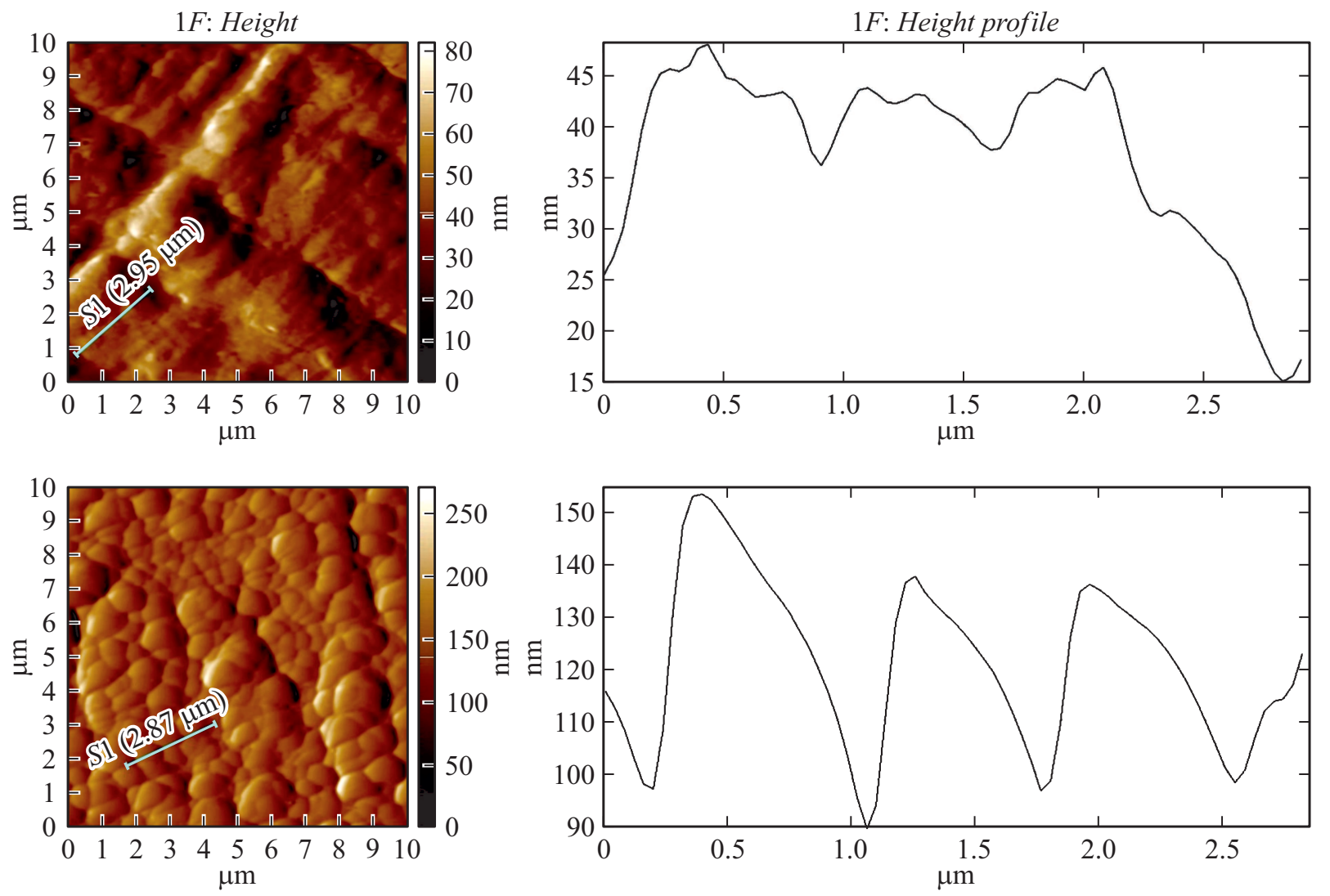

Рис. 3. Вверху слева - топографическое АСМ изображение образца $H$, доза $D=0.2 \mathrm{mGy,} \mathrm{поле} 10 \times 10 \mu \mathrm{m}$; справа - профиль поверхности, серии 2. Внизу - то же для образца $E_{h}$, доза $D=4500 \mathrm{mGy}$.
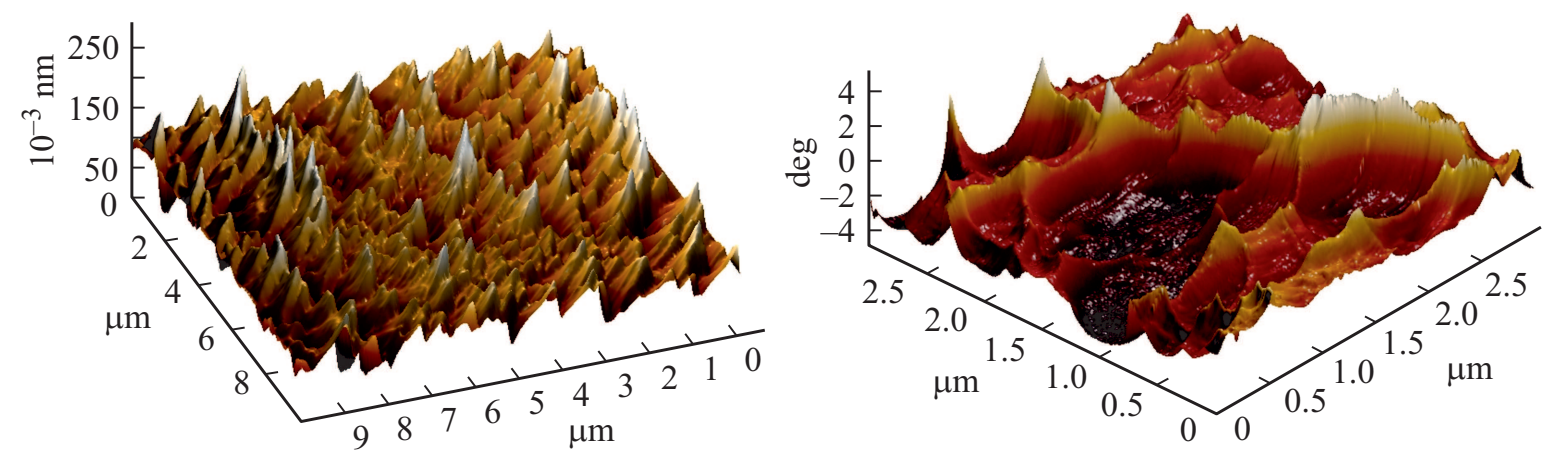

Рис. 4. АCM $3 D$-изображения образца $E_{h}$ серии 2 , доза $D=4500 \mathrm{mGy.} \mathrm{Слева} \mathrm{-} \mathrm{топографический} \mathrm{полуконтактный} \mathrm{режим,} \mathrm{поле}$ $10 \times 10 \mu \mathrm{m}$; справа - фазовый режим, поле $3 \times 3 \mu \mathrm{m}$.

ны облучения на расстояния $H-8.5 \mathrm{~cm}(D=0.2 \mathrm{mGy})$ и $E_{h}-1 \mathrm{~cm}(D=4500 \mathrm{mGy})$. Из сравнения профилей поверхности видно, что на отрезке $\sim 3 \mu$ m можно выделить три частицы размером около $0.7-0.9 \mu \mathrm{m}$. При совпадении латеральных размеров СЭ видно, что их высота у образца $E_{h}$ больше в 5 раз, чем у образца $H$. Глубина межструктурных впадин возросла с 0.0036 до $0.09 \mu \mathrm{m}$, а их форма изменилась от равноосной до клиновидной. Явно наблюдаемое при возрастании дозы углубление впадин хорошо объяснимо плазмохимическим воздействием кислорода на поверхность. Эффект, зависящий от концентрации кислородосодержащих молекул в газе, сильнее проявился на образце $E_{h}$, который облучался с плотностью потока $\sim 2 \cdot 10^{4} \mathrm{~s}^{-1} \cdot \mathrm{cm}^{-2}$, что на два порядка выше, чем у образца $H \sim 1 \cdot 10^{2} \mathrm{~s}^{-1} \cdot \mathrm{cm}^{-2}$.

Оценка усредненной величины шероховатости образцов $H$ и $E_{h}$ при обработке АCМ изображений показала с ростом дозы заметное возрастание от 0.044 до $0.175 \mu \mathrm{m}$. Этот эффект наглядно объясняется при переходе на $3 D$ изображение $10 \times 10 \mu \mathrm{m}$ и еще бо́льшее разрешение в фазовом режиме с полем $3 \times 3 \mu \mathrm{m}$. На рис. 4 слева видно, что на поверхности образца $E_{h}$, расположенного в 

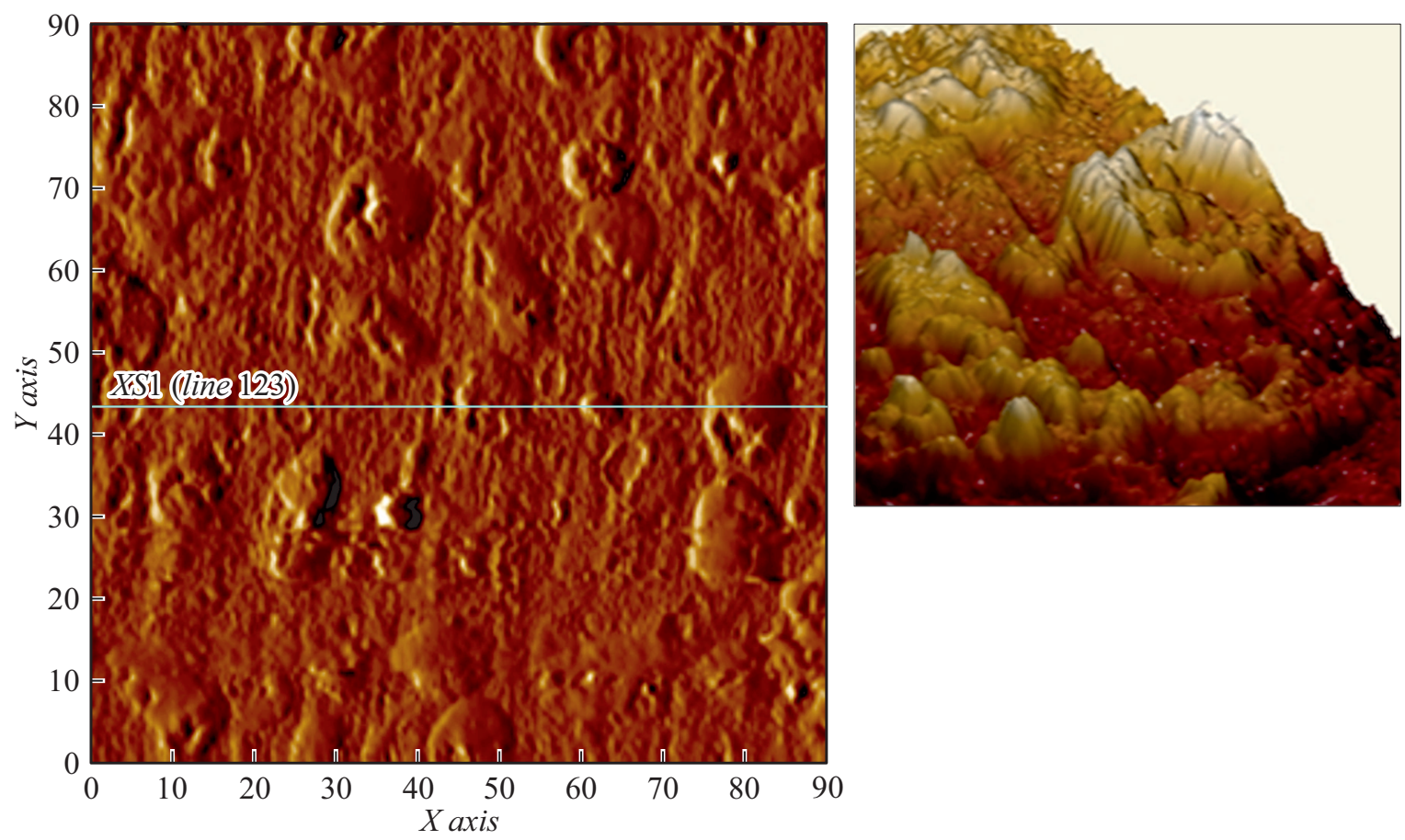

Рис. 5. АСМ изображение образца $E-H$, поле $90 \times 90 \mu \mathrm{m}$, серии 1 , доза $D=63 \mathrm{mGy}$, режим топографии; фрагмент справа $3 D$-изображения кратера, поле $10 \times 10 \mu \mathrm{m}$.

центре зоны облучения, образуется квазипериодический рельеф, представляющий собой чередование выступов и впадин. В режиме фазового контраста наблюдается ячеистый рельеф (рис. 4 справа). Ячейка представляет собой плоскую впадину с выпуклыми стенками. Более твердый, жесткий материал стенок, плохо поддающийся эрозии, на изображении фазы имеет более светлый оттенок.

Известно, что в ходе распыления в плазме газа на поверхности облученных пленок образуются локальные кластеры окислов, имеющие значительно более низкую скорость распыления материала, чем основа [16,17]. Окислы металлов могут играть роль собственных микромасок материала, приводящих к формированию системы микропиков на поверхности. Поэтому можно предположить, что физической причиной процесса формирования сильно выраженного микрорельефа с высотой микроструктур до $\sim 0.2 \mu \mathrm{m}$ (рис. 4) служит нуклеация оксидной фазы.

В результате облучения прототипов камер CSC глубина проникновения кислорода в фольгу составила около $10 \mu \mathrm{m}$, что привело в первой фазе окисления к образованию $\mathrm{Cu}_{2} \mathrm{O}$ и объясняет приведенные на рис. 3 и 4 эффекты [15].

В образцах серии 1, где аккумулированная доза в несколько раз выше, чем у образцов серии 2, дефектная структура проявляется более ярко. В этой серии более наглядны радиационные повреждения поверхности меди, наблюдается их эволюция с увеличением дозы облучения.

\section{2. Радиационные повреждения поверхности образцов}

На рис. 5 представлена поверхность образца $E-H$ серии 1 , набравшего дозу $D=63 \mathrm{mGy}$ рядом с центром зоны облучения (рис. $1, Y=3.5 \mathrm{~cm}$ ). Видно, что медное покрытие подвержено сильной радиационной эрозии. Хорошо просматриваются крупные частично вскрытые блистеры и области шелушения - флекинга.

Кратеры, которые образуются при разрушении блистеров, способны стать источником токов эмиссии. Их края представляют собой острия, которые из-за процессов распыления с увеличением дозы становятся все тоныше (рис. 4) [9]. Подобные дефекты присутствуют на поверхности всех образцов этой серии и имеют разнообразные формы и размеры. При АСМ исследовании образцов были обнаружены наличие всех фаз образования, развития и разрушения кратеров.

В табл. 1 представлены результаты измерений геометрических параметров основных эрозионных дефектов поверхности, полученных при помощи программного модуля Image Analysis 3.5 в режиме Section Analysis [15].

Из анализа изображений АCM и данных табл. 1 видно, что размеры дефектов растут в зависимости от близости к зоне облучения. Так, диаметры блистеров возрастают от 1.5 до $3.23 \mu \mathrm{m}$, а высота — от 0.6 до $2.7 \mu \mathrm{m}$. Самые крупные блистеры, кратеры и глубокий флекинг $(1.8 \mu \mathrm{m})$ были обнаружены на поверхности образца $E-H(D=63 \mathrm{mGy})$.

Исключением из закономерности является образец $E_{f}$, набравший $D=11500 \mathrm{mGy,} \mathrm{в} \mathrm{котором} \mathrm{из-за} \mathrm{близ-}$ 
Таблица 1. Параметры эрозионных дефектов [15]

\begin{tabular}{|c|c|c|c|c|c|c|c|}
\hline \multirow{3}{*}{$\begin{array}{c}\text { Расстояние } \\
\text { от центра зоны } \\
\text { облучения, cm }\end{array}$} & \multirow{3}{*}{ Образцы } & \multicolumn{6}{|c|}{ Структурные параметры/среднее, $\mu \mathrm{m}$} \\
\hline & & \multicolumn{2}{|c|}{ Блистер } & \multicolumn{2}{|c|}{ Флекинг } & \multicolumn{2}{|c|}{ Кратеры } \\
\hline & & Диаметр & Высота & Ширина & Глубина & Диаметр & Глубина \\
\hline 8.5 & $H$ & 1.5 & 0.6 & 6.1 & 0.2 & 2.3 & 0.2 \\
\hline 5.2 & $E-D$ & 0.80 & 1.3 & - & - & 1.1 & 0.07 \\
\hline 3.5 & $E-H$ & 3.23 & 2.7 & 12.5 & 1.8 & 3.0 & 0.4 \\
\hline 1.0 & $E_{f}$ & 3.16 & 1.9 & - & - & 1.1 & 0.8 \\
\hline
\end{tabular}
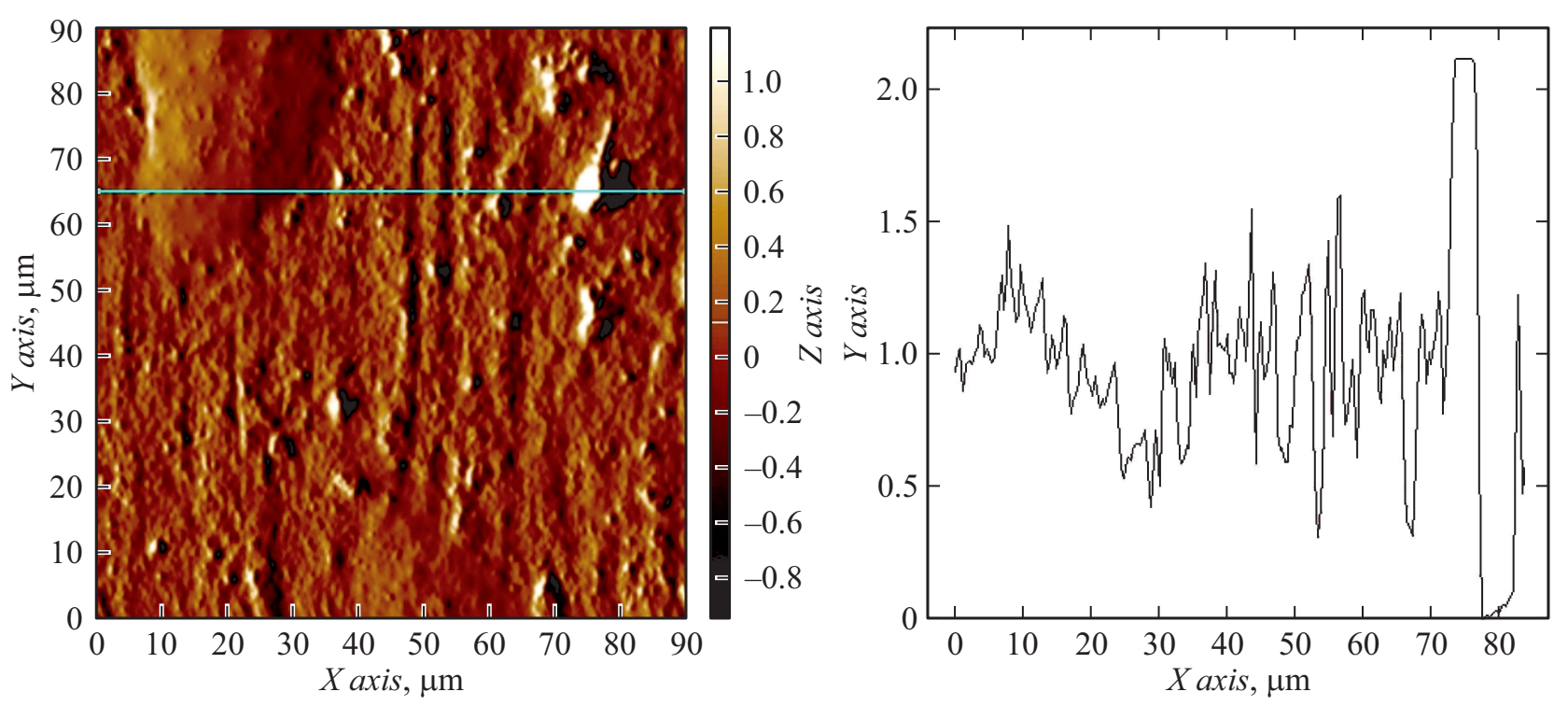

Рис. 6. АСМ изображение образца $E_{f}$, поле $90 \times 90 \mu \mathrm{m}$, серия 1 , доза $D=11500 \mathrm{mGy}$. Слева - режим топографии; справа профиль поверхности, измеренный вдоль линии сканирования.
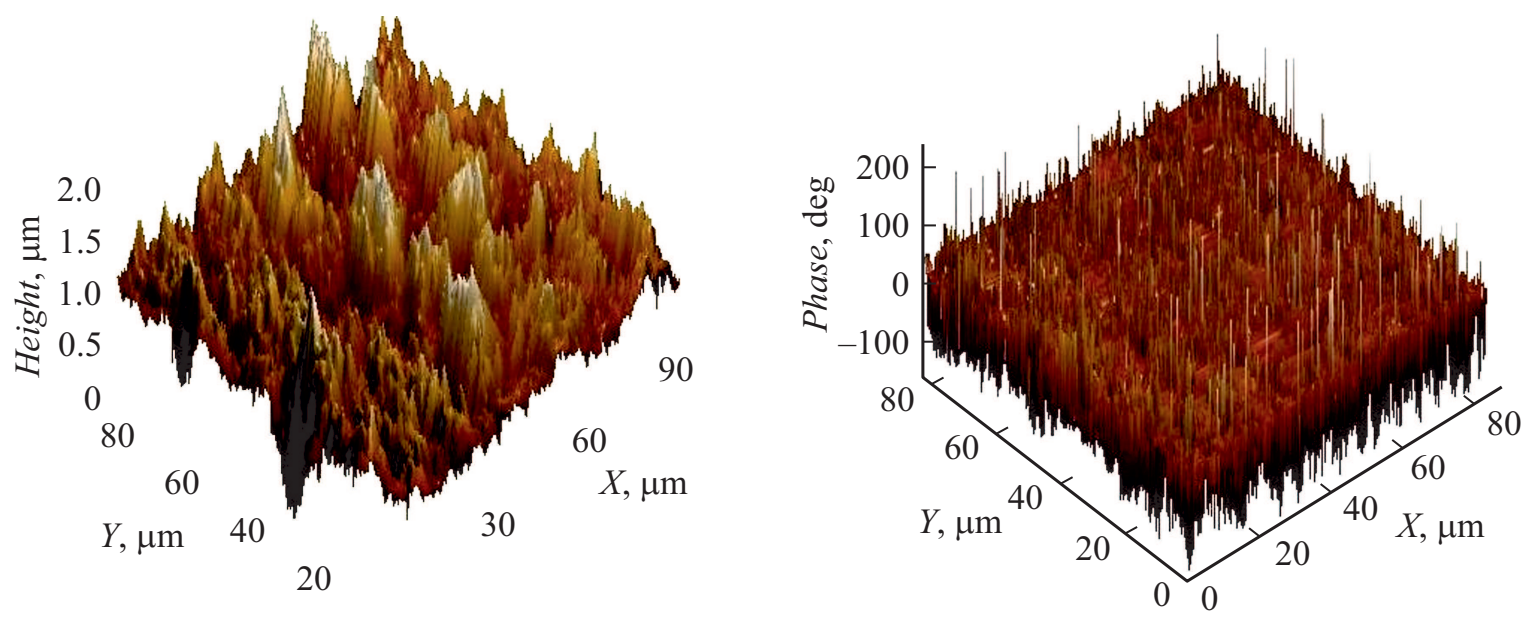

Рис. 7. АСМ $3 D$-изображения образца $E-H$, поле $90 \times 90 \mu \mathrm{m}$, серия 1 , доза $D=63 \mathrm{mGy.} \mathrm{Слева} \mathrm{-} \mathrm{режим} \mathrm{топографии;} \mathrm{справа} \mathrm{-}$ режим фазового контраста.

кого расположения к источнику произошло множество оплавлений поверхности, как видно на рис. 6. Здесь при максимальной глубине кратеров $0.8 \mu \mathrm{m}$ высота и диаметр блистеров в образце начинают уменьшаться.
Анализ поверхности образца $E_{f}$ с помощью Image Analysis 3.5 показал также, что для него характерна высокая равновесная пористость, мало подверженная блистерингу $[9,10]$. 
Таблица 2. Обработка АСМ изображений размером $90 \times 90 \mu \mathrm{m}$

\begin{tabular}{|c|c|c|c|c|c|}
\hline \multirow[b]{2}{*}{ Образцы } & \multicolumn{3}{|c|}{ Топография } & \multicolumn{2}{|c|}{ Фазовый контраст } \\
\hline & $\begin{array}{l}\text { Количество } \\
\text { микропиков }\end{array}$ & $\begin{array}{c}\text { Характерная } \\
\text { высота, } \mu \mathrm{m}\end{array}$ & $\begin{array}{c}\text { Поперечный } \\
\text { размер, } \mu \mathrm{m}\end{array}$ & $\begin{array}{l}\text { Количество } \\
\text { микропиков }\end{array}$ & $\begin{array}{l}\text { Поперечный } \\
\text { размер, } \mu \mathrm{m}\end{array}$ \\
\hline \multicolumn{6}{|c|}{ Серия 1} \\
\hline $\begin{array}{c}C \\
D \\
E-H \\
E_{f}\end{array}$ & $\begin{array}{l}200 \\
202 \\
351 \\
127\end{array}$ & $\begin{array}{l}1.1 \\
1.1 \\
1.1 \\
1.5\end{array}$ & $\begin{array}{l}1.0 \\
0.85 \\
0.65 \\
0.8\end{array}$ & $\begin{array}{l}286 \\
253 \\
382 \\
220\end{array}$ & $\begin{array}{l}1.0 \\
0.9 \\
0.55 \\
0.55\end{array}$ \\
\hline \multicolumn{6}{|c|}{ Серия 2} \\
\hline $\begin{array}{l}H \\
E_{h}\end{array}$ & $\begin{array}{l}252 \\
102\end{array}$ & $\begin{array}{l}0.57 \\
1.2\end{array}$ & $\begin{array}{c}- \\
1.3\end{array}$ & $\begin{array}{l}343 \\
296\end{array}$ & $\begin{array}{c}- \\
0.6\end{array}$ \\
\hline
\end{tabular}

Еще одной важной особенностью образцов, прошедших длительное облучение в ходе ресурсных испытаний, является появление включений новой фазы в виде тонких игловидных микропиков. Их появление и развитие хорошо видно на профиле поверхности (рис. 6). Видно, что микропики чаще всего формируются во впадинах в приграничных областях, а также на стыке зерен $(\sim 16 \mu \mathrm{m})$. Наличие подобных пиков может быть даже причиной микровзрывов на поверхности катода [7].

Наглядное подтверждение процесса формирования микропиков показано на рис. 7. Здесь приведены АСМ $3 D$-изображения $(90 \times 90 \mu \mathrm{m})$ образца $E-H$ серии 1 , удаленного от центра облучения на $3.5 \mathrm{~cm}$. Интересно, что плотность потока электронов при облучении образца $E-H$ в прототипе составила $F \approx 1 \cdot 10^{4} \mathrm{~s}^{-1} \cdot \mathrm{cm}^{-2}$, что сопоставимо с реальной загрузкой в пропорциональных камерах экспериментов CMS и LHCb на БАК. На рис. 7 слева в режиме АСМ топографии видно множество конусообразных микропиков высотой $\sim 2 \mu \mathrm{m}$.

При переходе в режим фазового контраста (рис. 7, справа), микропики видны в виде множества белых нитей. Данные обработки АСМ изображений $(90 \times 90 \mu \mathrm{m})$ шести образцов катодной поверхности приведены в табл. 2. Количественная оценка геометрии микропиков была выполнена при помощи штатной программы Grain Analysis C3M Solver Next „НТ-МДТ“.

В среднем высота микропиков составляет $\sim 1.2 \mu \mathrm{m}$. По данным табл. 2 в режиме фазового контраста их число почти в 1.5 раза больше, чем в топографическом режиме. По-видимому, некоторые из образовавшихся микропиков можно зафиксировать только в фазовом режиме [18]. Количество подобных образований на поверхности варьируется от 200 до 382 в зависимости от расположения образца в прототипе. Подробное исследование этого типа дефектов представляет особый интерес и планируется к представлению в отдельной публикации.

\section{3. Исследование катода пропорциональной камеры мюонного детектора LHCb}

Возможность экстраполяции результатов лабораторных ресурсных исследований на полномасштабные камеры, работающие на БАК, является критерием актуальности представленной работы. С этой целью методами АСМ было проведено сравнительное исследование образцов катодных поверхностей, взятых из пропорциональной камеры, демонтированной из экспериментальной установки LHCb после 10 лет эксплуатации на БАК из-за появления МЭ (время работы $\sim 1 \cdot 10^{8} \mathrm{~s}$, флюенс $\left.\sim 1 \cdot 10^{13} \mathrm{~cm}^{-2}\right)$. В эксперименте эта камера облучалась по всей площади потоком частиц с плотностью $1 \cdot 10^{4}-1 \cdot 10^{5} \mathrm{~s}^{-1} \cdot \mathrm{cm}^{-2}$ и продувалась рабочей газовой смесью $\mathrm{Ar} / \mathrm{CO}_{2} / \mathrm{CF}_{4}$. Конструкционные материалы катода этой камеры идентичны материалам пропорциональных камер CSC. Отработанные ранее АСМ методики и подходы к интерпретации изображений были применены к анализу поверхности двух образцов катода пропорциональной камеры. Первый из них был взят из зоны, где не наблюдался Мальтер-эффект (обозначен № 2). Второй образец извлечен с той же катодной плоскости, но из зоны, где Мальтер-эффект наблюдался (обозначен № 5). Расстояние между этими зонами на катоде составило $\sim 30 \mathrm{~cm}$.

Сравнение образцов на рис. 8 демонстрирует, что морфологически поверхность образца № 5 (МЭ) сходна с поверхностью образца $E_{f}$ лабораторной серии 1 на рис. 6. Поверхность образца № 5 мелкодисперсная, рыхлая и имеет высокую пористость (рис. 8, $a$ ). Белые точки на изображении - вершины микропиков. Поверхность образца № 2 (без МЭ) на рис. 8, $b$ подобна поверхности образца $E-D$ лабораторной серии 1 - рис. $8, c$ ). Волокнистость текстуры поверхности присутствует в обоих образцах, однако степень повреждения образцов из отработавшего на БАК детектора выше: наблюдаются 
a

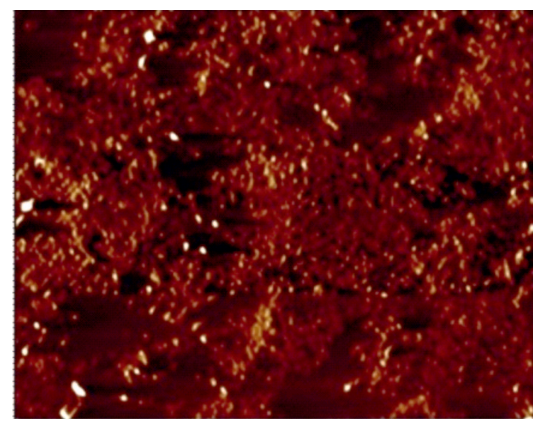

b
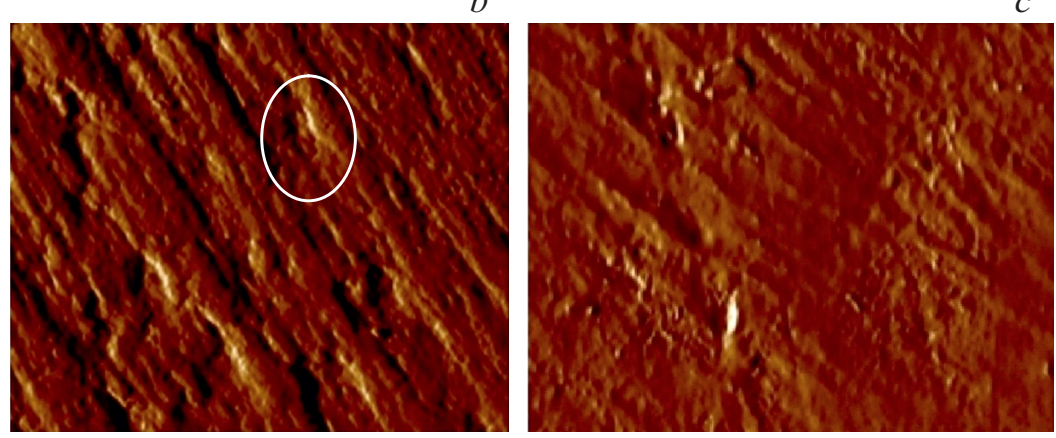

Рис. 8. АСМ топографические изображения, поле $90 \times 90 \mu \mathrm{m}: a-$ образец № 5, зона, где наблюдался МЭ; $b$ - образец № 2 , зона без МЭ; $c$ - образец $E-D$ серия 1 , доза $D=14 \mathrm{mGy}$.
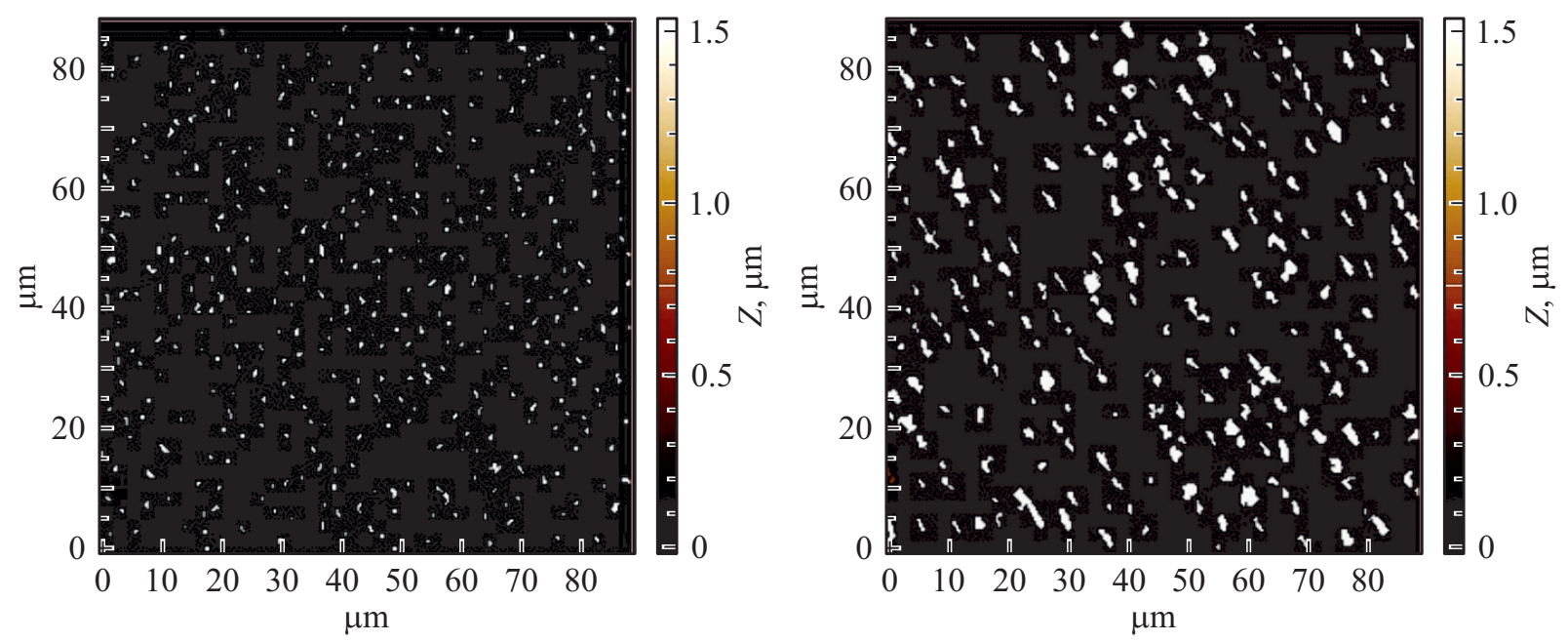

Рис. 9. Результаты анализа высоты микропиков на АСМ изображениях, поле $90 \times 90 \mu \mathrm{m}: a$ - образец № 5 из зоны, где наблюдался МЭ; $b$ - образец № 2 из зоны, где нет МЭ.

Таблица 3. Результаты обработки АСМ изображений размером $90 \times 90 \mu \mathrm{m}$

\begin{tabular}{c|c|c|c|c|c}
\hline \multirow{2}{*}{ Образцы } & \multicolumn{3}{|c|}{ Топография } & \multicolumn{3}{c}{ Фазовый контраст } \\
\cline { 2 - 6 } & $\begin{array}{c}\text { Количество } \\
\text { микропиков }\end{array}$ & $\begin{array}{c}\text { Характерная } \\
\text { высота, } \mu \mathrm{m}\end{array}$ & $\begin{array}{c}\text { Поперечный } \\
\text { размер, } \mu \mathrm{m}\end{array}$ & $\begin{array}{c}\text { Количество } \\
\text { микропиков }\end{array}$ & $\begin{array}{c}\text { Поперечный } \\
\text { размер, } \mu \mathrm{m}\end{array}$ \\
\hline № 2 & 230 & 1.1 & 1.2 & 286 & 0.4 \\
№ 5 (МЭ) & 415 & 0.6 & 0.5 & 455 & 0.5 \\
$E-D$ & 122 & 2.3 & 0.6 & 295 & 0.35
\end{tabular}

крупные кратеры со сложным рельефом дна, которые чередуются с террасными структурами и с участками, где гребни волокон сглажены.

Анализ изображений образца № 5 на рис. 8, $a$ и № 2 на рис. $8, b$ при помощи программного модуля Image Analysis 3.5. позволил выявить существенные различия в структуре и характере дефектности для зоны с МЭ и без него. Результаты анализа профиля поверхности образцов № 2 и № 5 представлен в табл. 3. Хорошо видно превышение по высоте у микропиков образца № 2. Их число и высота на поверхности образцов сопоставимы с данными для лабораторных образцов (табл. 2). В образце № 5 с МЭ количество пиков почти вдвое больше, а латеральные размеры и регистрируемая высота ниже. В основном в этом образце микропики расположены во впадинах, на стыках и по границам зерен (рис. 8,a). В образце № 2 характер расположения пиков иной: пики формируются на гребнях волокон (рис. $8, b)$. По-видимому, это может быть одной из причин более высоких значений высоты микропиков в образце без МЭ. Исходя из экспериментальных данных, можно предположить, что появление большого количества пиков в образце № 5 связано с другим механизмом их формирования. 
На рис. 9 показаны результаты измерения высот микропиков. Видно, что высота микропиков у образца № 2 (справа) систематически больше, чем у образца № 5 . Кроме того, они имеют протяженный характер, напоминающий гребни кратеров.

Таким образом, анализ характера дефектности поверхности образцов катода, полученных из пропорциональной камеры после работы на БАК, продемонстрировал его сходство с изменениями структуры поверхности в образцах после лабораторных ресурсных испытаний. Отличием стала степень синергетического эффекта воздействия плазмы и облучения на медную фольгу.

Сравнение дефектов образцов № 2 и № 5 позволяет предположить, что возникновение МЭ в пропорциональных камерах на БАК не связано с ростом высоты микропиков на меди и обусловлено плазмохимическим формированием на поверхности локализованных зон с низкой проводимостью.

\section{Заключение}

Применение АСМ методов для анализа образцов катодной поверхности прототипа многопроволочной пропорциональной камеры CSC позволило детально наблюдать эволюцию повреждений, вызванных длительным облучением медной фольги электронами ${ }^{90} \mathrm{Sr}$ в диапазоне доз от долей милигрей до десятков грей.

Сравнение образцов катода, взятых из пропорциональных камер, работавших на БАК, и из состаренных лабораторных прототипов, показало сходный характер дефектности. Это наблюдение, выполненное с помощью АCM, подтверждает актуальность лабораторных ресурсных испытаний, проводимых при исследовании пропорциональных камер.

Анализ АСМ изображений с использованием программного модуля Image Analysis 3.5 позволил уточнить характер повреждений поверхности меди, при которых возможно появление мальтеровского тока в детекторе. Таким образом, появляется возможность разработки превентивных методов, позволяющих подавить появление этого эффекта.

\section{Благодарности}

Авторы выражают искреннюю благодарность сотрудникам Научно-исследовательского Института Экспериментальной Физики (г. Саров) за помощь в проведении исследований. При их участии было проведено скрупулезное исследование образцов методами сканирующей силовой микроскопии. Авторы глубоко благодарны К.Н. Ермакову, ведущему научному сотруднику Петербургского института ядерной физики НИЦ „Курчатовский институт“ и В.М. Вахтелю, доценту Воронежского государственного университета, за внимательное прочтение и ценные рекомендации по содержанию статьи.

\section{Конфликт интересов}

Авторы заявляют, что у них нет конфликта интересов.

\section{Список литературы}

[1] T. Ferguson, G. Gavrilov, A. Korytov, A. Krivchitch, E. Kuznetsova, E. Lobachev, G. Mitselmakher, L. Schipunov. Nucl. Instrum. and Methods Phys. Res. A, 488, 240 (2002).

[2] O. Prokofev, D. Acosta, G. Apollinari, K. Arisaka, N. Bondar, R. Breedon, A. Bujak, B. Bylsma, N. Chester, R. Clare, D. Cline, R. Cousins, T. Cox, S. Dolinsky, S. Durkin, P. Eartly David, Yu. Ivanov, T. Ferguson, G. Gavrilov, J. Gilmore, J. Gu, L. Gutay, F. Hann, R. Hartmut, J. Hauser, K. He, M. Ignatenko, S. Ilie, C. Jiang, W. Ko, A. Korytov, A. Krivshich, E. Kuznetsova, J. Layter, R. Lee, P. Levchenko, T.Y. Ling., D. Loveless, S. Lusin, K. Maeshima, A. Madorsky, C. Matthey, M. Matveev, M. von der Mey, Guenakh Mitselmakher, S. Otwinowski, Padley B. Paul, I. Pal, Yu. Pishchalnikov, V. Razmyslovich, D. Reeder, J. Roberts, L. Shchipunov, I. Smirnov, V. Suvorov, J. Stasko, H. Sun, V. Sytnik, N. Terentev, M. Tripathi, S. Vavilov, A. Volkov, A. Vorobov, I. Vorobov, L. Wang, V. Yarba, L. Zhou, Z. Zhu. Nucl. Instrum. Methods Phys. Res. A, 515, 226 (2003). DOI: 10.1016/j.nima.2003.09.002

[3] E.P. Albicocco, L. Anderlini, M. Anellia, F. Archilli, G. Auriemma, W. Baldini, G. Bencivenni, N. Bondar, B. Bochin, D. Brundu, S. Cadeddu, P. Campana, G. Carboni, A. Cardini, M. Carletti, L. Casu, A. Chubykin, P. Ciambrone, E. Dané, P. De Simone, M. Fontana, P. Fresch, M. Gatta, G. Gavrilov, S. Gets, G. Graziani, A. Kashchuk, M. Korolev, S. Kotriakhova, E. Kuznetsova, A. Lai, O. Levitskaya, A. Loi, O. Maev, D. Maysuzenko, G. Martellotti, S. Nasybulin, P. Neustroev, R.G.C. Oldeman, M. Palutan, G. Passaleva, G. Penso, D. Pinci, R. Santacesaria, M. Santimaria, E. Santovetti, B. Saitta, A. Saputi, A. Sarti, C. Satriano, A. Satta, B. Schmidt, T. Schneider, B. Sciascia, A. Sciubba, R. Vazquez-Gomez, S. Vecchi, A. Vorobyev. JINST, 14, 11031 (2019). DOI: 10.1088/1748-0221/14/11/P11031.

[4] J.W. McConkey, C.P. Malone, P.V. Johnson, C. Winstead, V. McKoy, I. Kanik. Phys. Rep., 466, 1 (2008). DOI: 10.1016/j.physrep.2008.05.001

[5] С.А. Салтыков. Стереометрическая металлограбия (Металлургия, М., 1970)

[6] M. Flora Li, R. Waddingham, W.I. Milne, A.J. Flewitt, S. Speakman, J. Dutson, S. Wakeham, M. Thwaites. Thin Solid Films, 520, 1278 (2011). http://dx.doi.org/10.1016/j.tsf.2011.04.192.

[7] Г.А. Месяц. Взрывная электронная эмиссия (Физматлит, M., 2011) c. 30.

[8] R.E. Hurley, P.J. Dooley. J. Phys. D: Appl. Phys., 10, 195 (1977).

[9] В.А. Блейхер, В.П. Кривобоков. Эрозия поверхности твердого тела под действием мочных пучков заряженных частии (Наука, Новосибирск, 2014)

[10] S.B. Fisher. Radiat. Eff. Defect. S., 7, 173 (1971).

[11] М.И. Гусева, Ю.В. Мартыненко. УФН, 135 (4), 671 (1981).

[12] Н.Б. Волков, А.Е. Майер. ЖТФ, 72 (8), 34 (2002).

[13] H.H. Hansen. Appl. Radiat. Isotopes, 34, 1241 (1983). 
[14] S. Agostinelli, J. Allison, K. Amako, J. Apostolakis, H. Araujo, P. Arce, M. Asai, D. Axen, S. Banerjee, G. Barrand, Behner, L. Bellagamba, J. Boudreau, L. Broglia, A. Brunengo, H. Burkhardt, S. Chauvie, J. Chuma, R. Chytracek, G. Cooperman, G. Cosmo, P. Degtyarenko, A. Dell'Acqua, G. Depaola, D. Dietrich, R. Enami, A. Feliceiello, C. Ferguson, H. Fecefeldt, G. Folger, F. Foppiano, A. Forti, S. Garelli, S. Giani, R. Giannitrapani, D. Gibin, J.J. Gomes, V. Grichine, A. Grossheim, S. Guatelli, P. Gumplinger, R. Hamatsu, K. Hashimoto, H. Hasui, A. Heikkinen, A. Howard, V. Ivanchenko, A. Johnson, F.W. Jones, J. Kallenbach, N. Kanaya, M. Kawabata, Y. Kawabata, M. Kawaguti, S. Kelner, P. Kent, A. Kimura, T. Kodama, R. Kokoulin, M. Kossov, H. Kurashige, E. Lamanna, T. Lampen, V. Lara, V. Lefebure, F. Lei, M. Liendl, W. Lockman, F. Longo, S. Magni, M. Maire, E. Medernach, K. Minamimoto, de Freitas P. Mora, Y. Morita, K. Murakami, M. Nagamatu, R. Nartallo, P. Nieminen, T. Nishimura, K. Otsubo, M. Okamura, S. O’Neale, Y. Oohata, K. Paech, J. Perl, A. Pfeiffer, M.G. Pia, F. Ranjard, A. Rybin, S. Sadilov, E. Di Salvo, E. Santin, T. Sasaki, N. Savvas, Y. Sawada, S. Scherer, S. Sai, V. Sirotenko, O. Smith, N. Starkov, H. Stoeker, J. Sulkimo, M. Takahata, S. Tanaka, E. Tcherniaev, E. Tehrani Safai, M. Tropeano, P. Truscott, H. Uno, L. Urban, P. Urban, M. Verderi, A. Walkden, W. Wander, H. Weber, J.P. Wellisch, T. Wenaus, D.C. Williams, D. Wright, T. Yamada, H. Yoshida, D. Zschiesche. Nucl. Instrum. Methods Phys. Res. A, 506, 250 (2003).

[15] M.E. Buzoveria, N.V. Zavyalov, I.A. Karpov, M.I. Tkachenko, A. Dziuba, D.A. Maisuzenko, G.E. Gavrilov, S.A. Nasybulin. Phys. Atom. Nucl., 82 (9), 1252 (2019).

DOI: $10.1134 /$ S1063778819090035

[16] С.П. Зимин, Е.С. Горлачев, И.И. Амиров. Изв. вузов. Физика, 1/2, 269 (2011).

[17] А.И. Стадниченко, Ф.М. Сорокин, А.И. Боронин. ЖСХ, 9 (2), 341 (2008).

[18] В.Л. Миронов. Основы сканирующей зондовой микроскопии. Учебное пособие для студентов старших курсов высших учебных заведений (РАН. Институт физики микроструктур, Н. Новгород, 2004) 